JOURNAL OF

FUNCTION SPACES AND APPLICATIONS

Volume 3, Number 2 (2005), 163-181 (c) 2005, Scientific Horizon

http://www.jfsa.net

\title{
The random Wigner distribution of Gaussian stochastic processes with covariance in $S_{0}\left(\mathbb{R}^{2 d}\right)$
}

\author{
Patrik Wahlberg \\ (Communicated by Hans Feichtinger)
}

2000 Mathematics Subject Classification. 42B35, 60G15, 60G35, 62M15, $94 \mathrm{~A} 12$.

Keywords and phrases. Time-frequency analysis, the Feichtinger algebra, Gaussian scalar-valued stochastic processes, Wigner distribution, Wigner spectrum, Cohen's class.

\footnotetext{
Abstract. The paper treats time-frequency analysis of scalar-valued zero mean Gaussian stochastic processes on $\mathbb{R}^{d}$. We prove that if the covariance function belongs to the Feichtinger algebra $S_{0}\left(\mathbb{R}^{2 d}\right)$ then: (i) the Wigner distribution and the ambiguity function of the process exist as finite variance stochastic Riemann integrals, each of which defines a stochastic process on $\mathbb{R}^{2 d}$, (ii) these stochastic processes on $\mathbb{R}^{2 d}$ are Fourier transform pairs in a certain sense, and (iii) Cohen's class, ie convolution of the Wigner process by a deterministic function $\Phi \in C\left(\mathbb{R}^{2 d}\right)$, gives a finite variance process, and if $\Phi \in S_{0}\left(\mathbb{R}^{2 d}\right)$ then $W * \Phi$ can be expressed multiplicatively in the Fourier domain.
}

\section{Introduction}

For functions and distributions defined on $\mathbb{R}^{d}$ it is well known under what circumstances it is possible to define the Wigner distribution and the ambiguity function [7]. This paper concerns the corresponding conditions 
164 The random Wigner distribution of Gaussian stochastic processes

when the function or distribution is replaced by a zero mean Gaussian scalarvalued stochastic process. Suppose the covariance function $r \in S_{0}\left(\mathbb{R}^{2 d}\right)$. Then we prove that the Wigner distribution $W(t, \xi)$ and the ambiguity function $A(\theta, \tau)$ exist as stochastic Riemann integrals of finite variance. Moreover $W$ and $A$ constitute a stochastic Fourier integral transform pair in a certain sense. We are also interested in Cohen's class of time-frequency representations, ie the stochastic convolution integral $W * \Phi(t, \xi)$ where $\Phi$ is a deterministic kernel function. If $r \in S_{0}\left(\mathbb{R}^{2 d}\right)$ and $\Phi \in C\left(\mathbb{R}^{2 d}\right)$, we prove that $W * \Phi(t, \xi)$ is a finite variance process. If moreover $\Phi \in S_{0}\left(\mathbb{R}^{2 d}\right)$ then $W * \Phi(t, \xi)$ can be expressed as a stochastic Fourier integral of the process $A$ times the Fourier transform $\widehat{\Phi}$. The reformulation of the stochastic convolution integral is useful for optimization of $\Phi$ when $W * \Phi$ is used for estimation of $E(W)$. This idea is due to Sayeed and Jones [18].

We denote by $\int$ Riemann integrals, by $\int^{L}$ Lebesgue integrals, by $L^{p}\left(\mathbb{R}^{d}\right)$ the Lebesgue spaces, by $R\left(\mathbb{R}^{d}\right)$ the set of Riemann integrable functions over $\mathbb{R}^{d}$, by $A\left(\mathbb{R}^{d}\right)$ the Fourier algebra, by $\mathcal{S}\left(\mathbb{R}^{d}\right)$ the Schwartz space of smooth rapidly decreasing functions, by $C\left(\mathbb{R}^{d}\right)$ the set of continuous functions which are bounded in the supremum norm, and by $\widetilde{f}(t):=f(-t)$ the coordinate reflection operator. We will often use the Fourier Riemann integral $\mathcal{F}$, defined for functions $f \in L^{1}\left(\mathbb{R}^{d}\right) \cap C\left(\mathbb{R}^{d}\right)$ by

$$
(\mathcal{F} f)(\xi):=\widehat{f}(\xi):=\int_{\mathbb{R}^{d}} f(t) e^{-i\langle t, \xi\rangle} d t
$$

where $\langle t, \xi\rangle=\sum_{j=1}^{d} t_{j} \xi_{j}$. If $\widehat{f} \in L^{1}\left(\mathbb{R}^{d}\right) \cap C\left(\mathbb{R}^{d}\right)$ the inverse Fourier Riemann integral is defined by

$$
\left(\mathcal{F}^{-1} \widehat{f}\right)(t):=(2 \pi)^{-d} \int_{\mathbb{R}^{d}} \widehat{f}(\xi) e^{i\langle t, \xi\rangle} d \xi
$$

The assumptions on $f$ and $\widehat{f}$ implies that the Riemann integrals (1) and (2) can be replaced by Lebesgue integrals since the Lebesgue and Riemann integrals coincide for functions in $L^{1}\left(\mathbb{R}^{d}\right) \cap C\left(\mathbb{R}^{d}\right)$. For a function $f$ of several sets of variables we will denote partial Fourier integral with respect to variable sets indexed by $j, k$, by $\widehat{f}_{j, k}$. Suppose $f: \mathbb{R}^{d+m} \mapsto \mathbb{C}$ and $f(\cdot, x) \in L^{1}\left(\mathbb{R}^{d}\right) \cap C\left(\mathbb{R}^{d}\right)$ for all $x \in \mathbb{R}^{m}$. The partial Fourier Riemann integral in the first variable is defined by

$$
\left(\mathcal{F}_{1} f\right)(\xi, x):=\widehat{f}_{1}(\xi, x):=\int_{\mathbb{R}^{d}} f(t, x) e^{-i\langle t, \xi\rangle} d t, \xi \in \mathbb{R}^{d}, x \in \mathbb{R}^{m}
$$


We are interested in function spaces on which the Fourier Riemann integral is invertible in the uniform norm, ie $\left(\mathcal{F}^{-1} \circ \mathcal{F} f\right)(t)=f(t) \forall t \in \mathbb{R}^{d}$. We shall make extensive use of the function space known as Feichtinger's algebra $[2,3,7]$, often denoted $S_{0}\left(\mathbb{R}^{d}\right)$. Its definition can be based on the short-time Fourier transform (STFT). The STFT of a function $f: \mathbb{R}^{d} \mapsto \mathbb{C}$ with respect to a window function $g$ is defined by the Lebesgue integral

$$
V_{g} f(t, \xi):=\int_{\mathbb{R}^{d}}^{L} f(x) \overline{g(x-t)} e^{-i\langle\xi, x\rangle} d x
$$

and the Feichtinger algebra is defined by

$$
S_{0}\left(\mathbb{R}^{d}\right):=\left\{f \in L^{2}\left(\mathbb{R}^{d}\right):\|f\|_{S_{0}\left(\mathbb{R}^{d}\right)}:=\left\|V_{g} f\right\|_{L^{1}\left(\mathbb{R}^{2 d}\right)}<\infty\right\}
$$

for a fixed $g \in \mathcal{S}\left(\mathbb{R}^{d}\right)$. It is a Banach space which contains the Fréchet space $\mathcal{S}\left(\mathbb{R}^{d}\right), S_{0}\left(\mathbb{R}^{d}\right) \subset L^{1}\left(\mathbb{R}^{d}\right) \cap A\left(\mathbb{R}^{d}\right)$ and hence $S_{0}\left(\mathbb{R}^{d}\right) \subset C\left(\mathbb{R}^{d}\right)$. Different windows $g \in \mathcal{S}\left(\mathbb{R}^{d}\right)$ yield equivalent $S_{0}$ norms, and the admissible class of windows can be generalized to $g \in S_{0}\left(\mathbb{R}^{d}\right)$. The space $S_{0}\left(\mathbb{R}^{d}\right)$ is invariant under coordinate transformations and conjugation. It is a Banach algebra under both pointwise multiplication,

$$
\left\|f_{1} \cdot f_{2}\right\|_{S_{0}\left(\mathbb{R}^{d}\right)} \leq C_{1}\left\|f_{1}\right\|_{S_{0}\left(\mathbb{R}^{d}\right)}\left\|f_{2}\right\|_{S_{0}\left(\mathbb{R}^{d}\right)}
$$

and convolution,

$$
\left\|f_{1} * f_{2}\right\|_{S_{0}\left(\mathbb{R}^{d}\right)} \leq C_{2}\left\|f_{1}\right\|_{S_{0}\left(\mathbb{R}^{d}\right)}\left\|f_{2}\right\|_{S_{0}\left(\mathbb{R}^{d}\right)}
$$

for all $f_{1}, f_{2} \in S_{0}\left(\mathbb{R}^{d}\right), C_{1}, C_{2}>0$. The Fourier transform, defined by (1) using the Lebesgue integral, is for a proper choice of $g$ an isometry of $S_{0}\left(\mathbb{R}^{d}\right)$ onto $S_{0}\left(\widehat{\mathbb{R}^{d}}\right)$, where $\widehat{\mathbb{R}^{d}}=\mathbb{R}^{d}$ denotes the dual group of $\mathbb{R}^{d}$,

$$
\|\mathcal{F} f\|_{S_{0}\left(\mathbb{R}^{d}\right)}=\|f\|_{S_{0}\left(\mathbb{R}^{d}\right)} \forall f \in S_{0}\left(\mathbb{R}^{d}\right) .
$$

This isometry holds also if the Lebesgue integral in (1) is replaced by the Riemann integral. Due to $S_{0}\left(\mathbb{R}^{d}\right) \subset L^{1}\left(\mathbb{R}^{d}\right) \cap A\left(\mathbb{R}^{d}\right)$ we have [17]

$$
\left(\mathcal{F}^{-1} \circ \mathcal{F} f\right)(t)=f(t) \quad \forall t \in \mathbb{R}^{d} \forall f \in S_{0}\left(\mathbb{R}^{d}\right),
$$

where $\mathcal{F}$ is defined by the Lebesgue integral (1) and $\mathcal{F}^{-1}$ is defined by the Lebesgue integral (2). Again Lebesgue integrals can be replaced by Riemann integrals. The following two properties are proved in [2]. Restriction of an $S_{0}$ function is an $S_{0}$ function,

$$
f \in S_{0}\left(\mathbb{R}^{d+m}\right) \Longrightarrow f(\cdot, y) \in S_{0}\left(\mathbb{R}^{d}\right) \quad \forall y \in \mathbb{R}^{m},
$$


166 The random Wigner distribution of Gaussian stochastic processes

and partial Fourier Lebesgue integral of an $S_{0}$ function gives an $S_{0}$ function,

$$
f(x, y) \in S_{0}\left(\mathbb{R}^{d+m}\right) \Longrightarrow \mathcal{F}_{1} f(\xi, y) \in S_{0}\left(\mathbb{R}^{d+m}\right) .
$$

The integral in (11) can be replaced by a Riemann integral due to (10) and the fact that the Lebesgue and Riemann integrals are equal on $S_{0}\left(\mathbb{R}^{d}\right)$.

For another aspect of harmonic analysis of stochastic processes the space $S_{0}(G)$, where $G$ is a locally compact abelian group, has been proved to be useful as a test function space for generalized stochastic processes [10, 11].

\section{Second order stochastic processes on $\mathbb{R}^{d}$}

We study stochastic processes indexed by $t \in \mathbb{R}^{d}$ and denoted $X(t)$, or $X(t, \omega)$ when the dependence of $\omega \in \Omega$, the probability space, is of interest [1]. In applications $t$ often denotes time and then $d=1$, but in order to maintain generality we allow integer $d>1$ in which case the stochastic process is often called a stochastic field. A stochastic process or field is a mapping $X(t): \mathbb{R}^{d} \ni t \mapsto \mathcal{X}(\mathbb{P})$, where $\mathcal{X}(\mathbb{P})$ is the set of all $\mathbb{C}$-valued stochastic variables over a probability space $\Omega$ with probability measure $\mathbb{P}, \mathbb{P}(\Omega)=1$. The expectation ( $d \mathbb{P}$ integral) of the stochastic variable $X \in \mathcal{X}(\mathbb{P})$ is denoted $E(X)=\int_{\Omega} X(\omega) d \mathbb{P}(\omega)$. We denote by $L^{2}(\mathbb{P}):=\left\{X \in \mathcal{X}(\mathbb{P}) ; E|X|^{2}<\infty\right\}$ the Hilbert space of finite variance stochastic variables with scalar product $\langle X, Y\rangle_{L^{2}(\mathbb{P})}=E(X \bar{Y})$. A sequence $X_{n} \in L^{2}(\mathbb{P}), n=1,2, \ldots$, which converges in the $L^{2}(\mathbb{P})$ norm to $X \in L^{2}(\mathbb{P})$ is said to converge in mean square. This is denoted $X=\operatorname{li}_{n \rightarrow \infty}$ i. $X_{n}$. A process $X: \mathbb{R}^{d} \mapsto L^{2}(\mathbb{P})$ is called a second order process.

The autocovariance function $r$ of the process $X(t)$ is a function $r: \mathbb{R}^{2 d} \mapsto \mathbb{C}$ defined by

$$
r(t, s):=E(X(t) \overline{X(s)}),
$$

which is finite everywhere due to the Cauchy-Schwartz inequality. Several computations in this paper will involve the following fourth order moment function.

\section{Definition 2.1 .}

$$
c\left(t_{1}, \tau_{1}, t_{2}, \tau_{2}\right)=E\left(X\left(t_{1}+\tau_{1} / 2\right) \overline{X\left(t_{1}-\tau_{1} / 2\right)} \overline{X\left(t_{2}+\tau_{2} / 2\right)} X\left(t_{2}-\tau_{2} / 2\right)\right) .
$$

2.1. Gaussian processes. We restrict to $\mathbb{R}$ - or $\mathbb{C}$-valued processes $X(t)$ that have zero mean, $E(X(t))=0 \forall t \in \mathbb{R}^{d}$, and are Gaussian, ie $\left\{X\left(t_{j}\right)\right\}_{j=1}^{N}$ have multidimensional Gaussian probability distribution for any 
finite set $\left\{t_{j}\right\}_{j=1}^{N}[12,15]$. In the case of $\mathbb{C}$-valued processes we require the following property.

Definition 2.2. A circularly symmetric process is a zero mean $\mathbb{C}$ valued process $X(t)$ such that the processes $\left\{e^{i \theta} X(t)\right\}_{\theta \in[0,2 \pi)}$ are identically distributed for all $\theta \in[0,2 \pi)$.

According to Grettenberg's theorem [6, 15] circular symmetry is equivalent to

$$
E(X(t) X(s))=0 \quad \forall(t, s) \in \mathbb{R}^{2 d} .
$$

Let $x_{1}, x_{2}, x_{3}, x_{4}$ be zero mean real-valued Gaussian stochastic variables. Then Isserlis' theorem [8] p 244 states that

(15)

$$
E\left(x_{1} x_{2} x_{3} x_{4}\right)=E\left(x_{1} x_{2}\right) E\left(x_{3} x_{4}\right)+E\left(x_{1} x_{3}\right) E\left(x_{2} x_{4}\right)+E\left(x_{1} x_{4}\right) E\left(x_{2} x_{3}\right) .
$$

The formula (15) can be extended to zero mean complex-valued Gaussian stochastic variables by straight-forward computation. Thus for circularly symmetric Gaussian processes we have

$$
\begin{aligned}
E\left(X\left(t_{1}\right) \overline{X\left(t_{2}\right)} \overline{X\left(t_{3}\right)} X\left(t_{4}\right)\right)= & E\left(X\left(t_{1}\right) \overline{X\left(t_{2}\right)}\right) E\left(X\left(t_{4}\right) \overline{X\left(t_{3}\right)}\right) \\
& +E\left(X\left(t_{1}\right) \overline{X\left(t_{3}\right)}\right) E\left(X\left(t_{4}\right) \overline{X\left(t_{2}\right)}\right)
\end{aligned}
$$

since the last term of (15) vanishes due to (14). Using (16) and (12) Definition 2.1 can now be written

$$
c\left(t_{1}, \tau_{1}, t_{2}, \tau_{2}\right)=\left(r \otimes \bar{r} \circ \kappa+r \otimes \bar{r} \circ p_{2,3} \circ \kappa\right)\left(t_{1}, \tau_{1}, t_{2}, \tau_{2}\right)
$$

where $\kappa$ denotes the $\mathbb{R}^{4 d}$ linear isometric coordinate transformation

$$
\kappa\left(t_{1}, \tau_{1}, t_{2}, \tau_{2}\right)=\left(t_{1}+\tau_{1} / 2, t_{1}-\tau_{1} / 2, t_{2}+\tau_{2} / 2, t_{2}-\tau_{2} / 2\right),
$$

and $p_{2,3}$ denotes the coordinate transposition $p_{2,3}\left(t_{1}, \tau_{1}, t_{2}, \tau_{2}\right)=$ $\left(t_{1}, t_{2}, \tau_{1}, \tau_{2}\right)$. For a real-valued Gaussian process (15) gives

(19) $c\left(t_{1}, \tau_{1}, t_{2}, \tau_{2}\right)=\left(r \otimes r \circ \kappa+r \otimes r \circ p_{2,3} \circ \kappa+r \otimes r \circ p_{2,3,4} \circ \kappa\right)\left(t_{1}, \tau_{1}, t_{2}, \tau_{2}\right)$

where $p_{2,3,4}\left(t_{1}, \tau_{1}, t_{2}, \tau_{2}\right)=\left(t_{1}, \tau_{2}, \tau_{1}, t_{2}\right)$ is a coordinate permutation.

From formulæ (17) and (19) it is clear that if $r$ is a member of a certain Banach function space $B\left(\mathbb{R}^{2 d}\right)$, then $c$ will be a member of $B\left(\mathbb{R}^{4 d}\right)$ provided $B\left(\mathbb{R}^{2 d}\right)$ is a linear space invariant under conjugation, tensorization and linear isometric coordinate transformations. These weak requirements certainly hold for the space $S_{0}\left(\mathbb{R}^{2 d}\right)$ [2]. We shall study the 
168 The random Wigner distribution of Gaussian stochastic processes

case $r \in S_{0}\left(\mathbb{R}^{2 d}\right)$. For $\mathbb{R}$-valued Gaussian processes and $\mathbb{C}$-valued circularly symmetric Gaussian processes we hence have

$$
r \in S_{0}\left(\mathbb{R}^{2 d}\right) \Longrightarrow c \in S_{0}\left(\mathbb{R}^{4 d}\right) .
$$

\section{Stochastic integrals}

We need to define two types of stochastic integrals $[1,12,15]$. The first concerns Riemann integration of Hilbert space valued functions on $\mathbb{R}^{d}$. The second concerns integration of $\mathbb{C}$-valued functions with respect to a Hilbert space valued measure defined on $\mathbb{R}^{d}$.

The first type of stochastic integral is needed in order to define the Wigner distribution and the ambiguity function for stochastic processes. We use the theory of Riemann integration of Hilbert space valued functions [9, 12]. In our case the Hilbert space is $L^{2}(\mathbb{P})$. We will use the following results. Let $X: \mathbb{R}^{d} \mapsto L^{2}(\mathbb{P})$ be a stochastic process. Let $T>0$ and $K_{T} \subset \mathbb{R}^{d}$ denote the cube that have all coordinate moduli $\leq T$. Let $P=\left\{K_{j}\right\}_{j=1}^{N}$ denote a partition, ie a finite class of disjoint rectangle sets such that $\cup_{j} K_{j}=K_{T}$, and let $t_{j} \in K_{j}$. Provided the rhs inner limit exists in $L^{2}(\mathbb{P})$ for a sequence of partitions $\left\{P_{n}\right\}_{n=1}^{\infty}$, independently of the choices $t_{j} \in K_{j}$, and the outer limit exists in $L^{2}(\mathbb{P})$, the stochastic Riemann integral is defined by [12]

$$
\int_{\mathbb{R}^{d}} X(t) d t:=\underset{T \rightarrow \infty}{\lim } \underset{n \rightarrow \infty}{\operatorname{l.j} \text { i.m. }} \sum_{j=1}^{N} X\left(t_{j}\right) d t\left(K_{j}\right) .
$$

The process $X(t)$ is stochastic Riemann integrable if and only if the ordinary Riemann integral

$$
\iint_{\mathbb{R}^{2 d}} E(X(t) \overline{X(s)}) d t d s
$$

exists finitely. Let $Z \in L^{2}(\mathbb{P})$ and let $X(t)$ be stochastic Riemann integrable. Then by the Cauchy-Schwartz inequality in $L^{2}(\mathbb{P})$ and $(21)$

$$
E\left(\int_{\mathbb{R}^{d}} X(t) d t \bar{Z}\right)=\int_{\mathbb{R}^{d}} E(X(t) \bar{Z}) d t .
$$

If we choose $Z(\omega) \equiv 1 \in L^{2}(\mathbb{P})$ we obtain

$$
E\left(\int_{\mathbb{R}^{d}} X(t) d t\right)=\int_{\mathbb{R}^{d}} E(X(t)) d t .
$$


If $X(t)$ is Riemann integrable over $\mathbb{R}^{d}$ and $Y(t)$ is Riemann integrable over $\mathbb{R}^{m}$, then

$$
E\left(\int_{\mathbb{R}^{d}} X(t) d t \overline{\int_{\mathbb{R}^{m}} Y(t) d t}\right)=\iint_{\mathbb{R}^{d+m}} E(X(t) \overline{Y(s)}) d t d s .
$$

Since the probability measure $\mathbb{P}$ is finite, $L^{2}(\mathbb{P})$ contains all $\mathbb{C}$-valued functions which are constant as a function of $\omega \in \Omega$. For such a function $X$ we have $\|X\|_{L^{2}(\mathbb{P})}^{2}=\int_{\Omega}|X(\omega)|^{2} d \mathbb{P}(\omega)=|X|^{2}$. Thus $\mathbb{C}$ can be considered as an isometrically embedded subspace of $L^{2}(\mathbb{P})$, and deterministic functions $f: \mathbb{R}^{d} \mapsto \mathbb{C}$ can be considered as second order stochastic processes. The integral (22) for a function $f$ considered as a process is finite if and only if $f \in R\left(\mathbb{R}^{d}\right)$, since $E(f(t) \overline{f(s)})=f(t) \overline{f(s)}$. Thus $f$ has a stochastic Riemann integral if and only if $f \in R\left(\mathbb{R}^{d}\right)$, and by (21) its value as ordinary Riemann integral equals its stochastic Riemann integral.

Next we turn to the second type of stochastic integral which concerns integration of $\mathbb{C}$-valued functions with respect to a measure taking values in $L^{2}(\mathbb{P})[1,12]$. In this case the measure is a countably additive set function $d Z: \mathcal{B}\left(\mathbb{R}^{d}\right) \mapsto L^{2}(\mathbb{P})$ where $\mathcal{B}\left(\mathbb{R}^{d}\right)$ denotes the Borel $\sigma$-algebra of $\mathbb{R}^{d}$. If $X(t)$ is a process whose covariance function $r$ is the Fourier-Stieltjes transform of a measure of bounded variation $d \widehat{r}(\xi, \eta)$ in the sense of

$$
r(t, s)=(2 \pi)^{-d} \iint_{\mathbb{R}^{2 d}} e^{i(\langle\xi, t\rangle-\langle\eta, s\rangle)} d \widehat{r}(\xi, \eta),
$$

then $X(t)$ is called strongly harmonizable $[12,16]$. There exists an equivalence between such a spectral representation of the covariance function and a spectral representation of the process as a stochastic integral

$$
X(t)=(2 \pi)^{-d} \int_{\mathbb{R}^{d}} e^{i\langle\xi, t\rangle} d Z(\xi)
$$

where $d Z: \mathcal{B}\left(\mathbb{R}^{d}\right) \mapsto L^{2}(\mathbb{P})$. The measure $d Z$ can be defined for rectangle sets by difference operators of a map $Z: \mathbb{R}^{d} \mapsto L^{2}(\mathbb{P})$, called the spectral process, and then extended to $\mathcal{B}\left(\mathbb{R}^{d}\right)$. In the following we specialize to the case $r \in S_{0}\left(\mathbb{R}^{2 d}\right)$. We see from (8) that (26) holds where $d \widehat{r}(\xi, \eta)=\widehat{r}(\xi, \eta) d \xi d \eta$ and $\widehat{r} \in S_{0}\left(\mathbb{R}^{2 d}\right)$. Thus $X(t)$ is strongly harmonizable. Consider $Y_{\xi}(t):=e^{-i\langle\xi, t\rangle} X(t)$ as a process $\mathbb{R}^{d} \ni t \mapsto L^{2}(\mathbb{P})$ indexed by $\xi \in \mathbb{R}^{d}$. The rhs integral of $(25)$ for the process $Y_{\xi}(t)$ at two 
170 The random Wigner distribution of Gaussian stochastic processes

indices $\xi$ and $\eta$ is

$$
\iint_{\mathbb{R}^{2 d}} E\left(Y_{\xi}(t) \overline{Y_{\eta}(s)}\right) d t d s=\iint_{\mathbb{R}^{2 d}} r(t, s) e^{i(\langle\eta, s\rangle-\langle\xi, t\rangle)} d t d s=(2 \pi)^{d} \widehat{r}(\xi, \eta)
$$

by (26). Since $\widehat{r} \in C\left(\mathbb{R}^{2 d}\right)$ the integral (22) for the process $Y_{\xi}(t)$ is finite. Therefore we have a well defined stochastic integral

$$
Z(\xi):=\int_{\mathbb{R}^{d}} e^{-i\langle\xi, t\rangle} X(t) d t \in L^{2}(\mathbb{P}) \quad \forall \xi \in \mathbb{R}^{d},
$$

and by (25) and (28)

$$
E(Z(\xi) \overline{Z(\eta)})=(2 \pi)^{d} \widehat{r}(\xi, \eta)
$$

Set $\tilde{Y}_{t}(\xi):=(2 \pi)^{-d} e^{i\langle\xi, t\rangle} Z(\xi)$, then $\widetilde{Y}_{t}(\xi)$ is a process $\mathbb{R}^{d} \ni \xi \mapsto L^{2}(\mathbb{P})$ indexed by $t \in \mathbb{R}^{d}$. The rhs integral of $(25)$ for $\widetilde{Y}_{t}(\xi)$ at two indices $t$ and $s$ is, using (30),

$$
(2 \pi)^{-d} \iint_{\mathbb{R}^{2 d}} \widehat{r}(\xi, \eta) e^{i(\langle\xi, t\rangle-\langle\eta, s\rangle)} d \xi d \eta=r(t, s)
$$

again by (26). Thus the integral $(22)$ for the process $\widetilde{Y}_{t}(\xi)$ exists finitely, and

$$
\widetilde{X}(t):=(2 \pi)^{-d} \int_{\mathbb{R}^{d}} e^{i\langle\xi, t\rangle} Z(\xi) d \xi \in L^{2}(\mathbb{P}) \quad \forall t \in \mathbb{R}^{d}
$$

By $(25)$ and (31) $E|\widetilde{X}(t)|^{2}=r(t, t) \forall t \in \mathbb{R}^{d}$. We have $\tilde{X}(t)=X(t)$ in $L^{2}(\mathbb{P}) \forall t \in \mathbb{R}^{d}$. In fact, by application of (23) twice we obtain

$$
E(\tilde{X}(t) \overline{X(t)})=(2 \pi)^{-d} \int_{\mathbb{R}^{d}}\left(\int_{\mathbb{R}^{d}} r(s, t) e^{-i\langle\xi, s\rangle} d s\right) e^{i\langle\xi, t\rangle} d \xi=r(t, t) \forall t \in \mathbb{R}^{d},
$$

by (10) and (9), since $r \in S_{0}\left(\mathbb{R}^{2 d}\right)$. Using $E|X(t)|^{2}=r(t, t)$ we thus have $E|X(t)-\tilde{X}(t)|^{2}=0$ for all $t \in \mathbb{R}^{d}$. According to (27) and (32) the spectral process of $X(t)$ can thus be defined by $(29)$.

\section{Time-frequency analysis of the covariance function}

The Wigner spectrum $[4,14,18]$ of a process $X(t)$ with covariance function $r$ is defined by 


$$
W_{E}(t, \xi):=\int_{\mathbb{R}^{d}} r(t+\tau / 2, t-\tau / 2) e^{-i\langle\tau, \xi\rangle} d \tau=\left(\mathcal{F}_{2} r \circ \kappa_{0}\right)(t, \xi),
$$

where $\kappa_{0}$ denotes the linear isometric $\mathbb{R}^{2 d}$ coordinate transformation $\kappa_{0}(t, \tau)=(t+\tau / 2, t-\tau / 2)$. The subscript $E$ is used to indicate that it involves expectation under the integral as opposed to the random Wigner process to be defined below. The Wigner spectrum is a generalization of the spectral measure of a weakly stationary process. In fact, for such processes there is a function $f$ such that $r(t, s)=f(t-s)$, so $W_{E}(t, \xi)=\widehat{f}(\xi)$ which by definition is the spectral measure of a weakly stationary process.

The expected ambiguity function of the process is defined by

$$
A_{E}(\theta, \tau):=\int_{\mathbb{R}^{d}} r(t+\tau / 2, t-\tau / 2) e^{-i\langle t, \theta\rangle} d t=\left(\mathcal{F}_{1} r \circ \kappa_{0}\right)(\theta, \tau) .
$$

If $r \in S_{0}\left(\mathbb{R}^{2 d}\right)$ then by (11) $W_{E}, A_{E} \in S_{0}\left(\mathbb{R}^{2 d}\right) . A_{E}$ and $W_{E}$ are Fourier transform pairs in the sense of

$$
\begin{aligned}
W_{E}(t, \xi) & =(2 \pi)^{-d} \iint_{\mathbb{R}^{2 d}} A_{E}(\theta, \tau) e^{i(\langle\theta, t\rangle-\langle\tau, \xi\rangle)} d \theta d \tau \\
& =\mathcal{F}_{1}^{-1} \mathcal{F}_{2} A_{E} \\
A_{E}(\theta, \tau) & =(2 \pi)^{-d} \iint_{\mathbb{R}^{2 d}} W_{E}(t, \xi) e^{i(\langle\tau, \xi\rangle-\langle\theta, t\rangle)} d t d \xi \\
& =\mathcal{F}_{1} \mathcal{F}_{2}^{-1} W_{E} .
\end{aligned}
$$

This is clear from $\mathcal{F}_{1} W_{E}=\mathcal{F}_{1} \mathcal{F}_{2} r \circ \kappa_{0}=\mathcal{F}_{2} \mathcal{F}_{1} r \circ \kappa_{0}=\mathcal{F}_{2} A_{E}$.

\section{Existence of second order Wigner and ambiguity processes}

In this section we prove that $r \in S_{0}\left(\mathbb{R}^{2 d}\right)$ for a Gaussian process is a sufficient condition for the existence of a stochastic Wigner distribution and a stochastic ambiguity function in $L^{2}(\mathbb{P})$. We define the Wigner process of a process $X(t)$ using the same formula as the definition of the Wigner distribution for deterministic functions and distributions [5, 7]. The Wigner process is hence defined by the stochastic Riemann integral [13, 14, 18]

$$
W(t, \xi):=\int_{\mathbb{R}^{d}} X(t+\tau / 2) \overline{X(t-\tau / 2)} e^{-i\langle\tau, \xi\rangle} d \tau,
$$

and the ambiguity process, analogously to the ambiguity function [7], is defined by the stochastic integral 
172 The random Wigner distribution of Gaussian stochastic processes

$$
A(\theta, \tau):=\int_{\mathbb{R}^{d}} X(t+\tau / 2) \overline{X(t-\tau / 2)} e^{-i\langle t, \theta\rangle} d t
$$

The processes $W$ and $A$ are thus maps $\mathbb{R}^{2 d} \mapsto \mathcal{X}(\mathbb{P})$. The process $W$ was first defined by Martin [13], who also gave a condition on the fourth order moment function $c$, sufficient for $W$ to have finite variance, namely the requirement that (39) should be finite. This condition is satisfied for a Gaussian stochastic process if $r \in S_{0}\left(\mathbb{R}^{2 d}\right)$ according to the next theorem.

Theorem 5.1. Suppose $r \in S_{0}\left(\mathbb{R}^{2 d}\right)$. Then we have $(i) W(t, \xi) \in L^{2}(\mathbb{P})$ and $E(W(t, \xi))=W_{E}(t, \xi) \forall(t, \xi) \in \mathbb{R}^{2 d}, W$ is a continuous process, and (ii) $A(\theta, \tau) \in L^{2}(\mathbb{P})$ and $E(A(\theta, \tau))=A_{E}(\theta, \tau) \forall(\theta, \tau) \in \mathbb{R}^{2 d}, A$ is a continuous process.

Proof. Consider $Y_{t, \xi}(\tau):=X(t+\tau / 2) \overline{X(t-\tau / 2)} e^{-i\langle\tau, \xi\rangle}$ as a process $\mathbb{R}^{d} \ni \tau \mapsto L^{2}(\mathbb{P})$ indexed by $(t, \xi) \in \mathbb{R}^{2 d}$. It is a second order process since according to $(20) c \in S_{0}\left(\mathbb{R}^{4 d}\right) \subset C\left(\mathbb{R}^{4 d}\right)$. Using Definition 2.1 the rhs integral of $(25)$ for this process at two indices $\left(t_{1}, \xi_{1}\right)$ and $\left(t_{2}, \xi_{2}\right)$ is

$$
\iint_{\mathbb{R}^{2 d}} c\left(t_{1}, \tau_{1}, t_{2}, \tau_{2}\right) e^{-i\left(\left\langle\tau_{1}, \xi_{1}\right\rangle-\left\langle\tau_{2}, \xi_{2}\right\rangle\right)} d \tau_{1} d \tau_{2}=\widehat{c}_{2,4}\left(t_{1}, \xi_{1}, t_{2},-\xi_{2}\right)
$$

which is finite because $\widehat{c}_{2,4} \in S_{0}\left(\mathbb{R}^{4 d}\right)$ by (11). Thus the integral corresponding to (22) for the process $Y_{t, \xi}(\tau)$ is finite and we conclude that $Y_{t, \xi}(\tau)$ is stochastic Riemann integrable over $\mathbb{R}^{d}$, ie by $(37) W(t, \xi) \in L^{2}(\mathbb{P})$ for all $(t, \xi) \in \mathbb{R}^{2 d}$. By (25) and (39)

$$
E\left(W\left(t_{1}, \xi_{1}\right) \overline{W\left(t_{2}, \xi_{2}\right)}\right)=\widehat{c}_{2,4}\left(t_{1}, \xi_{1}, t_{2},-\xi_{2}\right)
$$

The $L^{2}(\mathbb{P})$-continuity of $W(t, \xi)$ follows from the continuity of $\widehat{c}_{2,4}$ [12]. By $(24)$ and $(34), E(W(t, \xi))=W_{E}(t, \xi)$ for all $(t, \xi) \in \mathbb{R}^{2 d}$. Thus (i) is proved. The proof of (ii) is similar. Instead of (40) we now have

$$
E\left(A\left(\theta_{1}, \tau_{1}\right) \overline{A\left(\theta_{2}, \tau_{2}\right)}\right)=\widehat{c}_{1,3}\left(\theta_{1}, \tau_{1},-\theta_{2}, \tau_{2}\right) .
$$

\section{The Wigner and ambiguity processes as a Fourier transform pair}

If $r \in S_{0}\left(\mathbb{R}^{2 d}\right)$ the processes $W$ and $A$ is a Fourier transform pair in the following stochastic sense. This result is a generalization of the deterministic relations $(36)$. 
Theorem 6.1. Suppose $r \in S_{0}\left(\mathbb{R}^{2 d}\right)$. Then $W(t, \xi)$ and $A(\theta, \tau)$ defined by (37) and (38), respectively, is a stochastic Fourier transform pair in the sense of the following two stochastic integral identities in $L^{2}(\mathbb{P})$.

$$
\begin{aligned}
& W(t, \xi)=(2 \pi)^{-d} \iint_{\mathbb{R}^{2 d}} A(\theta, \tau) e^{i(\langle\theta, t\rangle-\langle\tau, \xi\rangle)} d \theta d \tau \quad \forall(t, \xi) \in \mathbb{R}^{2 d} \\
& A(\theta, \tau)=(2 \pi)^{-d} \iint_{\mathbb{R}^{2 d}} W(t, \xi) e^{i(\langle\tau, \xi\rangle-\langle\theta, t\rangle)} d t d \xi \quad \forall(\theta, \tau) \in \mathbb{R}^{2 d}
\end{aligned}
$$

Proof. Consider $Y_{t, \xi}(\theta, \tau):=(2 \pi)^{-d} A(\theta, \tau) e^{i(\langle\theta, t\rangle-\langle\tau, \xi\rangle)}$ as a process $\mathbb{R}^{2 d} \ni(\theta, \tau) \mapsto L^{2}(\mathbb{P})$ indexed by $(t, \xi) \in \mathbb{R}^{2 d}$. It is a second order process by Theorem 5.1 and (41). Using (41) the rhs integral of (25) for $Y_{t, \xi}(\theta, \tau)$ at two indices $\left(t_{1}, \xi_{1}\right)$ and $\left(t_{2}, \xi_{2}\right)$ is

$$
\begin{aligned}
& (2 \pi)^{-2 d} \iiint_{\mathbb{R}^{4 d}} \int \widehat{c}_{1,3}\left(\theta_{1}, \tau_{1},-\theta_{2}, \tau_{2}\right) e^{i\left(\left\langle\theta_{1}, t_{1}\right\rangle-\left\langle\theta_{2}, t_{2}\right\rangle-\left\langle\tau_{1}, \xi_{1}\right\rangle+\left\langle\tau_{2}, \xi_{2}\right\rangle\right)} d \theta_{1} d \theta_{2} d \tau_{1} d \tau_{2} \\
& =\widehat{c}_{2,4}\left(t_{1}, \xi_{1}, t_{2},-\xi_{2}\right)
\end{aligned}
$$

by (11), (10) and (9) since $c \in S_{0}\left(\mathbb{R}^{4 d}\right)$. Thus $Y_{t, \xi}(\theta, \tau)$ is stochastic integrable over $\mathbb{R}^{2 d}$ and denoting the rhs of $(42)$ by $\widetilde{W}(t, \xi)$ we have $\widetilde{W}(t, \xi) \in L^{2}(\mathbb{P})$ for all $(t, \xi) \in \mathbb{R}^{2 d}$. Let $(t, \xi) \in \mathbb{R}^{2 d}$ be arbitrary. By $(25)$ and (44)

$$
E|\widetilde{W}(t, \xi)|^{2}=\widehat{c}_{2,4}(t, \xi, t,-\xi) .
$$

By (25), (38), (23) and Definition 2.1

$$
\begin{aligned}
E(\widetilde{W}(t, \xi) \overline{W(t, \xi)})= & (2 \pi)^{-d} \iiint_{\mathbb{R}^{3 d}}\left(\int_{\mathbb{R}^{d}} c\left(t_{1}, \tau_{1}, t, \tau_{2}\right) e^{-i\left\langle\theta, t_{1}\right\rangle} d t_{1}\right) \\
& \cdot e^{i\left(\langle\theta, t\rangle-\left\langle\xi, \tau_{1}-\tau_{2}\right\rangle\right)} d \theta d \tau_{1} d \tau_{2}=\widehat{c}_{2,4}(t, \xi, t,-\xi)
\end{aligned}
$$

by (11), (10) and (9) since $c \in S_{0}\left(\mathbb{R}^{4 d}\right)$. From (40), (45) and (46) we obtain $E|\widetilde{W}(t, \xi)-W(t, \xi)|^{2}=0$ for all $(t, \xi) \in \mathbb{R}^{2 d}$. Hence (42) has been proved. The identity (43) is proved in a similar fashion.

Cohen's class (see $\S 8$ ), ie the stochastic convolution integral $W * \Phi$ where $\Phi$ is a deterministic function, can be used for estimation of the Wigner spectrum [18]. The following theorem shows that the squared $L^{2}(\mathbb{P})$ norm, integrated over $(t, \xi) \in \mathbb{R}^{2 d}$, equals the corresponding ambiguity 
174 The random Wigner distribution of Gaussian stochastic processes

domain integral. This facilitates optimization of the kernel $\Phi$ because the convolution is transformed into a multiplication in the ambiguity domain.

Theorem 6.2. Suppose $W_{1}(t, \xi)$ and $A_{1}(\theta, \tau)$ are two processes defined on $\mathbb{R}^{2 d}$ which are related by the stochastic Fourier integral

$$
W_{1}(t, \xi)=(2 \pi)^{-d} \iint_{\mathbb{R}^{2 d}} A_{1}(\theta, \tau) e^{i(\langle\theta, t\rangle-\langle\tau, \xi\rangle)} d \theta d \tau \quad \forall(t, \xi) \in \mathbb{R}^{2 d}
$$

and suppose $g\left(\theta_{1}, \tau_{1}, \theta_{2}, \tau_{2}\right):=E\left(A_{1}\left(\theta_{1}, \tau_{1}\right) \overline{A_{1}\left(\theta_{2}, \tau_{2}\right)}\right) \in S_{0}\left(\mathbb{R}^{4 d}\right)$. Then

$$
\iint_{\mathbb{R}^{2 d}} E\left|W_{1}(t, \xi)\right|^{2} d t d \xi=\iint_{\mathbb{R}^{2 d}} E\left|A_{1}(\theta, \tau)\right|^{2} d \theta d \tau<\infty .
$$

Proof. By (25)

$$
\begin{aligned}
E\left|W_{1}(t, \xi)\right|^{2}= & (2 \pi)^{-2 d} \iiint_{\mathbb{R}^{4 d}} \int g\left(\theta_{1}, \tau_{1}, \theta_{2}, \tau_{2}\right) e^{i\left(\left\langle\theta_{1}-\theta_{2}, t\right\rangle-\left\langle\tau_{1}-\tau_{2}, \xi\right\rangle\right)} \\
& \cdot d \theta_{1} d \theta_{2} d \tau_{1} d \tau_{2} \\
= & (2 \pi)^{-2 d} \iint_{\mathbb{R}^{2 d}}^{L} \widehat{g}_{3,4}\left(\theta_{1}, \tau_{1}, t,-\xi\right) e^{i\left(\left\langle\theta_{1}, t\right\rangle-\left\langle\tau_{1}, \xi\right\rangle\right)} d \theta_{1} d \tau_{1}
\end{aligned}
$$

by the Fubini theorem, since the integrand in the first integral belongs to $S_{0}\left(\mathbb{R}^{4 d}\right) \subset L^{1}\left(\mathbb{R}^{4 d}\right) \cap C\left(\mathbb{R}^{4 d}\right)$ and thus the Riemann integral can be replaced by the Lebesgue integral. By $(11) \widehat{g}_{3,4} \in S_{0}\left(\mathbb{R}^{4 d}\right) \subset L^{1}\left(\mathbb{R}^{4 d}\right)$. Thus we have $\iint_{\mathbb{R}^{2 d}}^{L} E\left|W_{1}(t, \xi)\right|^{2} d t d \xi<\infty$. Fubini's theorem, (9) and (10) now justifies

$$
\iint_{\mathbb{R}^{2 d}}^{L} E\left|W_{1}(t, \xi)\right|^{2} d t d \xi=\iint_{\mathbb{R}^{2 d}}^{L} g\left(\theta_{1}, \tau_{1}, \theta_{1}, \tau_{1}\right) d \theta_{1} d \tau_{1}=\iint_{\mathbb{R}^{2 d}}^{L} E\left|A_{1}(\theta, \tau)\right|^{2} d \theta d \tau .
$$

We define $h_{1}(t, \xi, x, y):=\widehat{g}(-t+x / 2, \xi+y / 2, t+x / 2,-\xi+y / 2) \in S_{0}\left(\mathbb{R}^{4 d}\right)$ by $(8)$, and $h_{2}(\theta, \tau, x, y):=g(\theta+x / 2, \tau+y / 2, \theta-x / 2, \tau-y / 2) \in S_{0}\left(\mathbb{R}^{4 d}\right)$. Then by (49) and (10), $E\left|W_{1}(t, \xi)\right|^{2}=(2 \pi)^{-2 d} h_{1}(t, \xi, 0,0) \in S_{0}\left(\mathbb{R}^{2 d}\right)$ and $E\left|A_{1}(\theta, \tau)\right|^{2}=h_{2}(\theta, \tau, 0,0) \in S_{0}\left(\mathbb{R}^{2 d}\right)$. Thus all integrals in (50) can be replaced by Riemann integrals.

Corollary 6.3. If $r \in S_{0}\left(\mathbb{R}^{2 d}\right)$ then

$$
\iint_{\mathbb{R}^{2 d}} E|W(t, \xi)|^{2} d t d \xi=\iint_{\mathbb{R}^{2 d}} E|A(\theta, \tau)|^{2} d \theta d \tau<\infty
$$


Proof. Use Theorem 6.1, (41), $\widehat{c}_{1,3} \in S_{0}\left(\mathbb{R}^{4 d}\right)$ and Theorem 6.2.

\section{Marginal Properties}

The next two theorems treat the stochastic marginal properties of $W(t, \xi)$. They are generalizations of the deterministic marginal properties of $W_{E}(t, \xi)$. If $r \in S_{0}\left(\mathbb{R}^{2 d}\right)$, the time marginal property for $W_{E}$ is, using $(34)$,

$$
(2 \pi)^{-d} \int_{\mathbb{R}^{d}} W_{E}(t, \xi) d \xi=\left(r \circ \kappa_{0}\right)(t, 0)=r(t, t)=E|X(t)|^{2} \quad \forall t \in \mathbb{R}^{d}
$$

by (11) and (9). Let us compute the frequency marginal property for $W_{E}$ when $r \in S_{0}\left(\mathbb{R}^{2 d}\right)$,

$$
\begin{aligned}
\int_{\mathbb{R}^{d}} W_{E}(t, \xi) d t & =\int_{\mathbb{R}^{d}}\left(\mathcal{F}_{2} r \circ \kappa_{0}\right)(t, \xi) d t=\left(\mathcal{F} r \circ \kappa_{0}\right)(0, \xi) \\
& =(\mathcal{F} r)\left(\kappa_{0}^{-t}(0, \xi)\right),
\end{aligned}
$$

where $\kappa_{0}^{-t}$ denotes the transpose of the inverse of $\kappa_{0}(t, \tau)=(t+\tau / 2, t-\tau / 2)$, ie $\kappa_{0}^{-t}(0, \xi)=(\xi,-\xi)$. From (26), where $d \widehat{r}(\xi, \eta)=\widehat{r}(\xi, \eta) d \xi d \eta$ and $\widehat{r} \in S_{0}\left(\mathbb{R}^{2 d}\right)$, we have $(\mathcal{F} r)(\xi,-\eta)=(2 \pi)^{d} \widehat{r}(\xi, \eta)$. Thus by (30) we obtain the frequency marginal

$$
\int_{\mathbb{R}^{d}} W_{E}(t, \xi) d t=(2 \pi)^{d} \widehat{r}(\xi, \xi)=E|Z(\xi)|^{2} \quad \forall \xi \in \mathbb{R}^{d}
$$

where $Z(\xi)$ is the spectral process defined by (29). We shall now generalize the formulas (52) and (54) to hold in a stochastic sense.

Theorem 7.1. If $r \in S_{0}\left(\mathbb{R}^{2 d}\right)$ then we have in $L^{2}(\mathbb{P})$ for all $t \in \mathbb{R}^{d}$

$$
(2 \pi)^{-d} \int_{\mathbb{R}^{d}} W(t, \xi) d \xi=|X(t)|^{2}
$$

Proof. By Theorem 5.1 and (40) the integral (22) corresponding to the process $\mathbb{R}^{d} \ni \xi \mapsto W(t, \xi), t$ fixed, is finite, since $\widehat{c}_{2,4} \in S_{0}\left(\mathbb{R}^{4 d}\right) \Rightarrow$ $\widehat{c}_{2,4}(t, \cdot, t, \cdot \cdot) \in S_{0}\left(\mathbb{R}^{2 d}\right) \subset R\left(\mathbb{R}^{2 d}\right) \forall t \in \mathbb{R}^{d}$ by (10). Thus the lhs of (55) exists in $L^{2}(\mathbb{P})$. By $(25)$ and $(40)$

$$
\begin{aligned}
E\left|(2 \pi)^{-d} \int_{\mathbb{R}^{d}} W(t, \xi) d \xi\right|^{2} & =(2 \pi)^{-2 d} \iint_{\mathbb{R}^{2 d}} \widehat{c}_{2,4}\left(t, \xi_{1}, t,-\xi_{2}\right) d \xi_{1} d \xi_{2} \\
& =c(t, 0, t, 0) \quad \forall t \in \mathbb{R}^{d}
\end{aligned}
$$


176 The random Wigner distribution of Gaussian stochastic processes

due to (10) and (9). By (23) applied twice, (37) and $|X(t)|^{2} \in L^{2}(\mathbb{P})$ which follows from $c \in S_{0}\left(\mathbb{R}^{4 d}\right)$,

$$
\begin{aligned}
E\left((2 \pi)^{-d} \int_{\mathbb{R}^{d}} W(t, \xi) d \xi|X(t)|^{2}\right) & =(2 \pi)^{-d} \int_{\mathbb{R}^{d}}\left(\int_{\mathbb{R}^{d}} c(t, \tau, t, 0) e^{-i\langle\xi, \tau\rangle} d \tau\right) d \xi \\
& =c(t, 0, t, 0) \quad \forall t \in \mathbb{R}^{d} .
\end{aligned}
$$

Since $E|X(t)|^{4}=c(t, 0, t, 0)$ by Definition 2.1 we conclude using (56) and (57) that $\left.\left.E\left|(2 \pi)^{-d} \int_{\mathbb{R}^{d}} W(t, \xi) d \xi-\right| X(t)\right|^{2}\right|^{2}=0 \quad \forall t \in \mathbb{R}^{d}$.

Theorem 7.2. If $r \in S_{0}\left(\mathbb{R}^{2 d}\right)$ then we have in $L^{2}(\mathbb{P})$ for all $\xi \in \mathbb{R}^{d}$

$$
\int_{\mathbb{R}^{d}} W(t, \xi) d t=|Z(\xi)|^{2}
$$

where $Z(\xi)$ is the spectral process defined by (29).

Proof. The integral (22) corresponding to the process $\mathbb{R}^{d} \ni t \mapsto W(t, \xi)$, $\xi$ fixed, is finite, again by Theorem 5.1 and (40), since $\widehat{c}_{2,4} \in S_{0}\left(\mathbb{R}^{4 d}\right) \Rightarrow$ $\widehat{c}_{2,4}(\cdot, \xi, \cdot \cdot,-\xi) \in S_{0}\left(\mathbb{R}^{2 d}\right) \subset R\left(\mathbb{R}^{2 d}\right) \forall \xi \in \mathbb{R}^{d}$ by (10). Thus the lhs of (58) exists in $L^{2}(\mathbb{P})$. By $(25)$

$$
\begin{aligned}
E\left|\int_{\mathbb{R}^{d}} W(t, \xi) d t\right|^{2} & =\iint_{\mathbb{R}^{2 d}} \widehat{c}_{2,4}\left(t_{1}, \xi, t_{2},-\xi\right) d t_{1} d t_{2} \\
& =\widehat{c}(0, \xi, 0,-\xi) \quad \forall \xi \in \mathbb{R}^{d} .
\end{aligned}
$$

Consider $Y_{\xi}(t, s):=X(t) \overline{X(s)} e^{i\langle\xi, s-t\rangle}$ as a process $\mathbb{R}^{2 d} \ni(t, s) \mapsto L^{2}(\mathbb{P})$ indexed by $\xi$. It is a second order process because $c \in S_{0}\left(\mathbb{R}^{4 d}\right)$. Using Definition 2.1 the integral (22) for the process is $Y_{\xi}(t, s)$

$$
\iiint_{\mathbb{R}^{4 d}} \int c\left(t_{1}, s_{1}, t_{2}, s_{2}\right) e^{-i\left\langle\xi, s_{1}-s_{2}\right\rangle} d t_{1} d t_{2} d s_{1} d s_{2}=\widehat{c}(0, \xi, 0,-\xi) .
$$

Thus $Y_{\xi}(t, s)$ is stochastic integrable over $\mathbb{R}^{2 d}$, and we have by (29)

$$
|Z(\xi)|^{2}=\iint_{\mathbb{R}^{2 d}} X(t) \overline{X(s)} e^{i\langle\xi, s-t\rangle} d t d s \in L^{2}(\mathbb{P}),
$$

and by (25) and (60)

$$
E|Z(\xi)|^{4}=\widehat{c}(0, \xi, 0,-\xi) \quad \forall \xi \in \mathbb{R}^{d} .
$$


By (25), (61), (37) and (23)

$$
\begin{aligned}
E\left(\int_{\mathbb{R}^{d}} W(t, \xi) d t|Z(\xi)|^{2}\right) & =\iiint_{\mathbb{R}^{3 d}}\left(\int_{\mathbb{R}^{d}} c(t, \tau, u, v) e^{-i\langle\xi, \tau\rangle} d \tau\right) e^{i\langle\xi, v\rangle} d t d v d u \\
& =\widehat{c}(0, \xi, 0,-\xi) \quad \forall \xi \in \mathbb{R}^{d}
\end{aligned}
$$

From (59), (62) and (63) we conclude $\left.\left.E\left|\int_{\mathbb{R}^{d}} W(t, \xi) d t-\right| Z(\xi)\right|^{2}\right|^{2}=0$ $\forall \xi \in \mathbb{R}^{d}$.

\section{Cohen's class for second order processes}

In the next theorem we show that the process $W(t, \xi)$ can be convolved with certain deterministic functions $\Phi(t, \xi)$, giving a finite variance process. The convolution is often called Cohen's class of time-frequency distributions $[4,7]$. If $\Phi \in S_{0}\left(\mathbb{R}^{2 d}\right)$ the convolution corresponds to multiplication of $A(\theta, \tau)$ with $\phi(\theta, \tau)=\mathcal{F}_{1} \mathcal{F}_{2}^{-1} \Phi$. Suppose $\Phi \in L^{1}\left(\mathbb{R}^{2 d}\right) \cap C\left(\mathbb{R}^{2 d}\right)$. Then the Fourier Riemann integral of $\Phi$ is well defined and we define

$$
\phi(\theta, \tau):=(2 \pi)^{-d} \iint_{\mathbb{R}^{2 d}} \Phi(t, \xi) e^{i(\langle\tau, \xi\rangle-\langle\theta, t\rangle)} d t d \xi .
$$

Theorem 8.1. Suppose $r \in S_{0}\left(\mathbb{R}^{2 d}\right)$. If $\Phi \in C\left(\mathbb{R}^{2 d}\right)$ then the convolution stochastic integral defined by

(65) $W * \Phi(t, \xi):=\iint_{\mathbb{R}^{2 d}} W(t-s, \xi-\eta) \Phi(s, \eta) d s d \eta \in L^{2}(\mathbb{P}) \quad \forall(t, \xi) \in \mathbb{R}^{2 d}$.

If $\Phi \in S_{0}\left(\mathbb{R}^{2 d}\right)$ we have in $L^{2}(\mathbb{P})$

$$
W * \Phi(t, \xi)=\iint_{\mathbb{R}^{2 d}} A(\theta, \tau) \phi(\theta, \tau) e^{i(\langle\theta, t\rangle-\langle\tau, \xi\rangle)} d \theta d \tau \quad \forall(t, \xi) \in \mathbb{R}^{2 d}
$$

$A(\theta, \tau) \phi(\theta, \tau)=(2 \pi)^{-2 d} \iint_{\mathbb{R}^{2 d}}(W * \Phi)(t, \xi) e^{i(\langle\tau, \xi\rangle-\langle\theta, t\rangle)} d t d \xi \quad \forall(\theta, \tau) \in \mathbb{R}^{2 d}$.

Proof. Suppose $\Phi \in C\left(\mathbb{R}^{2 d}\right)$. By Theorem 5.1, $Y_{t, \xi}(s, \eta):=W(t-s$, $\xi-\eta) \Phi(s, \eta)$ is a second order process $\mathbb{R}^{2 d} \ni(s, \eta) \mapsto L^{2}(\mathbb{P})$ indexed by $(t, \xi) \in \mathbb{R}^{2 d}$. The integrand corresponding to the integrand of (22) for the 
178 The random Wigner distribution of Gaussian stochastic processes

process $Y_{t, \xi}(s, \eta)$ is, using $(40)$,

$$
\begin{aligned}
E & \left(W\left(t-s_{1}, \xi-\eta_{1}\right) \Phi\left(s_{1}, \eta_{1}\right) \overline{W\left(t-s_{2}, \xi-\eta_{2}\right) \Phi\left(s_{2}, \eta_{2}\right)}\right) \\
& =\widehat{c}_{2,4}\left(t-s_{1}, \xi-\eta_{1}, t-s_{2},-\xi+\eta_{2}\right) \Phi\left(s_{1}, \eta_{1}\right) \overline{\Phi\left(s_{2}, \eta_{2}\right)} .
\end{aligned}
$$

Since $\widehat{c}_{2,4} \in S_{0}\left(\mathbb{R}^{4 d}\right) \subset L^{1}\left(\mathbb{R}^{4 d}\right) \cap C\left(\mathbb{R}^{4 d}\right)$ and $\Phi \otimes \bar{\Phi} \in C\left(\mathbb{R}^{4 d}\right)$, (68) is Riemann integrable as a function of $\left(s_{1}, \eta_{1}, s_{2}, \eta_{2}\right) \in \mathbb{R}^{4 d}$. We conclude that the integral $(22)$ of the process $Y_{t, \xi}(s, \eta)$ is finite and thus (65) is a well defined stochastic integral in $L^{2}(\mathbb{P})$.

Let $\Phi \in S_{0}\left(\mathbb{R}^{2 d}\right)$. Then (65) holds since $S_{0}\left(\mathbb{R}^{2 d}\right) \subset C\left(\mathbb{R}^{2 d}\right)$, and by (8) $\phi \in S_{0}\left(\mathbb{R}^{2 d}\right)$. From (25) and (68) we have

$$
\begin{aligned}
E( & \left.W * \Phi\left(t_{1}, \xi_{1}\right) \overline{W * \Phi\left(t_{2}, \xi_{2}\right)}\right) \\
= & \iiint_{\mathbb{R}^{4 d}} \int_{c_{2,4}}\left(t_{1}-s_{1}, \xi_{1}-\eta_{1}, t_{2}-s_{2},-\xi_{2}+\eta_{2}\right) \\
& \times \Phi\left(s_{1}, \eta_{1}\right) \overline{\Phi\left(s_{2}, \eta_{2}\right)} d s_{1} d s_{2} d \eta_{1} d \eta_{2} \\
= & \widehat{c}_{2,4} * \Psi\left(t_{1}, \xi_{1}, t_{2},-\xi_{2}\right)
\end{aligned}
$$

where $\Psi\left(s_{1}, \eta_{1}, s_{2}, \eta_{2}\right)=\Phi\left(s_{1}, \eta_{1}\right) \overline{\Phi\left(s_{2},-\eta_{2}\right)} \in S_{0}\left(\mathbb{R}^{4 d}\right)$. Using (64) we can write

$$
(2 \pi)^{-2 d} \mathcal{F} \Psi\left(\theta_{1},-\tau_{1},-\theta_{2},-\tau_{2}\right)=\phi\left(\theta_{1}, \tau_{1}\right) \overline{\phi\left(\theta_{2}, \tau_{2}\right)} .
$$

Since by $(7) \widehat{c}_{2,4} * \Psi \in S_{0}\left(\mathbb{R}^{4 d}\right)$ we have from (9), (69) and (70)

$$
\begin{aligned}
E|W * \Phi(t, \xi)|^{2}= & (2 \pi)^{-4 d} \iiint_{\mathbb{R}^{4 d}} \int_{\mathcal{F}} \widehat{c}_{2,4}\left(\theta_{1}, \tau_{1}, \theta_{2}, \tau_{2}\right) \mathcal{F} \Psi\left(\theta_{1}, \tau_{1}, \theta_{2}, \tau_{2}\right) \\
& \cdot e^{i\left(\left\langle t, \theta_{1}+\theta_{2}\right\rangle+\left\langle\xi, \tau_{1}-\tau_{2}\right\rangle\right)} d \theta_{1} d \theta_{2} d \tau_{1} d \tau_{2} \\
= & \iiint_{\mathbb{R}^{4 d}} \int \widehat{c}_{1,3}\left(\theta_{1}, \tau_{1},-\theta_{2}, \tau_{2}\right) \phi\left(\theta_{1}, \tau_{1}\right) \overline{\phi\left(\theta_{2}, \tau_{2}\right)} \\
& \cdot e^{i\left(\left\langle t, \theta_{1}-\theta_{2}\right\rangle-\left\langle\xi, \tau_{1}-\tau_{2}\right\rangle\right)} d \theta_{1} d \theta_{2} d \tau_{1} d \tau_{2} \quad \forall(t, \xi) \in \mathbb{R}^{2 d}
\end{aligned}
$$

We now turn to the integrand of $(66) . \widetilde{Y}_{t, \xi}(\theta, \tau):=A(\theta, \tau) \phi(\theta, \tau) e^{i(\langle\theta, t\rangle-\langle\tau, \xi\rangle)}$ is by Theorem 5.1 and $(41)$ a second order process $\mathbb{R}^{2 d} \ni(\theta, \tau) \mapsto L^{2}(\mathbb{P})$ indexed by $(t, \xi) \in \mathbb{R}^{2 d}$. The integral (22) corresponding to the process 
$\widetilde{Y}_{t, \xi}(\theta, \tau)$ is by $(41)$

$$
\begin{aligned}
& \iiint_{\mathbb{R}^{4 d}} \int_{\widehat{c}_{1,3}}\left(\theta_{1}, \tau_{1},-\theta_{2}, \tau_{2}\right) \phi\left(\theta_{1}, \tau_{1}\right) \overline{\phi\left(\theta_{2}, \tau_{2}\right)} e^{i\left(\left\langle t, \theta_{1}-\theta_{2}\right\rangle-\left\langle\xi, \tau_{1}-\tau_{2}\right\rangle\right)} d \theta_{1} d \theta_{2} d \tau_{1} d \tau_{2} \\
& \quad=E|W * \Phi(t, \xi)|^{2} .
\end{aligned}
$$

We conclude that the rhs of $(66)$ defines a stochastic integral in $L^{2}(\mathbb{P})$ which we denote $\widetilde{W}(t, \xi)$. By $(25)$ and $(72) E|\widetilde{W}(t, \xi)|^{2}=E|W * \Phi(t, \xi)|^{2}$. By (25), (37) and (38)

$$
E\left(W(t-s, \xi-\eta) \overline{A\left(\theta_{2}, \tau_{2}\right)}\right)=\widehat{c}_{2,3}\left(t-s, \xi-\eta,-\theta_{2}, \tau_{2}\right) \quad \forall(t, \xi) \in \mathbb{R}^{2 d}
$$

and thus by application of (25) once again, followed by (64)

$$
\begin{aligned}
E(W * \Phi(t, \xi) \overline{\widetilde{W}(t, \xi)})= & \iiint_{\mathbb{R}^{4 d}} \int_{\widehat{c}_{2,3}}\left(t_{1}, \eta_{1},-\theta_{2}, \tau_{2}\right) \Phi\left(t-t_{1}, \xi-\eta_{1}\right) \overline{\phi\left(\theta_{2}, \tau_{2}\right)} \\
& \cdot e^{i\left(\left\langle\xi, \tau_{2}\right\rangle-\left\langle t, \theta_{2}\right\rangle\right)} d t_{1} d \eta_{1} d \tau_{2} d \theta_{2} \\
= & \iiint \int_{\mathbb{R}^{4 d}} \widehat{c}_{1,3}\left(\theta_{1}, \tau_{1},-\theta_{2}, \tau_{2}\right) \phi\left(\theta_{1}, \tau_{1}\right) \overline{\phi\left(\theta_{2}, \tau_{2}\right)} \\
& \cdot e^{i\left(\left\langle t, \theta_{1}-\theta_{2}\right\rangle-\left\langle\xi, \tau_{1}-\tau_{2}\right\rangle\right)} d \theta_{1} d \theta_{2} d \tau_{1} d \tau_{2} \forall(t, \xi) \in \mathbb{R}^{2 d} .
\end{aligned}
$$

Now (71), $E|\widetilde{W}(t, \xi)|^{2}=E|W * \Phi(t, \xi)|^{2}$ and (74) gives $E \mid \widetilde{W}(t, \xi)-W *$ $\left.\Phi(t, \xi)\right|^{2}=0 \forall(t, \xi) \in \mathbb{R}^{2 d}$ and (66) is proved.

We proceed to prove $(67) . Y_{\tau, \theta}^{\prime}(t, \xi):=(2 \pi)^{-2 d}(W * \Phi)(t, \xi) e^{i(\langle\tau, \xi\rangle-\langle\theta, t\rangle)}$ is a process $\mathbb{R}^{2 d} \ni(t, \xi) \mapsto L^{2}(\mathbb{P})$ indexed by $(\tau, \theta) \in \mathbb{R}^{2 d}$. It is a second order process since (69) is finite, which is a consequence of $\Psi, \widehat{c}_{2,4} \in S_{0}\left(\mathbb{R}^{4 d}\right)$ and (7). Using (69) and (70) we have

$$
\begin{aligned}
& \iiint_{\mathbb{R}^{4 d}} \int_{1} E\left(W * \Phi\left(t_{1}, \xi_{1}\right) \overline{W * \Phi\left(t_{2}, \xi_{2}\right)}\right) \\
& \times e^{i\left(\left\langle\tau, \xi_{1}-\xi_{2}\right\rangle-\left\langle\theta, t_{1}-t_{2}\right\rangle\right)} d t_{1} d t_{2} d \xi_{1} d \xi_{2} \\
& \quad=\mathcal{F} \widehat{c}_{2,4}(\theta,-\tau,-\theta,-\tau) \cdot \mathcal{F} \Psi(\theta,-\tau,-\theta,-\tau) \\
& \quad=(2 \pi)^{4 d} \widehat{c}_{1,3}(\theta, \tau,-\theta, \tau)|\phi(\theta, \tau)|^{2} .
\end{aligned}
$$


180 The random Wigner distribution of Gaussian stochastic processes

Since $\widehat{c}_{1,3} \in S_{0}\left(\mathbb{R}^{4 d}\right)$ and $\phi \in S_{0}\left(\mathbb{R}^{2 d}\right)$ (75) is finite. Hence the integral (22) for the process $Y_{\tau, \theta}^{\prime}(t, \xi)$ is finite and the rhs of $(67)$ is a well defined stochastic integral in $L^{2}(\mathbb{P})$. We denote it $\widetilde{A}(\theta, \tau)$. By $(25)$ and $(75)$

$$
E|\widetilde{A}(\theta, \tau)|^{2}=\widehat{c}_{1,3}(\theta, \tau,-\theta, \tau)|\phi(\theta, \tau)|^{2} .
$$

Denoting $g\left(\theta_{1}, \tau_{1}\right):=\widehat{c}_{1,3}\left(\theta_{1}, \tau_{1},-\theta, \tau\right) \phi\left(\theta_{1}, \tau_{1}\right)$, we have by $(25),(23),(65)$, (37), (38) and (64)

$$
\begin{aligned}
& E(W * \Phi(t, \xi) \overline{A(\theta, \tau)})=\iint_{\mathbb{R}^{2 d}} \widehat{c}_{2,3}(t-s, \xi-\eta,-\theta, \tau) \Phi(s, \eta) d s d \eta \\
& \quad=\iint_{\mathbb{R}^{2 d}} \widehat{c}_{1,3}\left(\theta_{1}, \tau_{1},-\theta, \tau\right) \phi\left(\theta_{1}, \tau_{1}\right) e^{i\left(\left\langle\theta_{1}, t\right\rangle-\left\langle\tau_{1}, \xi\right\rangle\right)} d \theta_{1} d \tau_{1} \\
& =\widehat{g}(-t, \xi) .
\end{aligned}
$$

By (10) and (6) $g \in S_{0}\left(\mathbb{R}^{2 d}\right)$ which implies $\widehat{g} \in S_{0}\left(\mathbb{R}^{2 d}\right)$. Using (77), (23) and (9) we obtain

$$
\begin{aligned}
E(\widetilde{A}(\theta, \tau) \overline{A(\theta, \tau)}) & =(2 \pi)^{-2 d} \iint_{\mathbb{R}^{2 d}} \widehat{g}(-t, \xi) e^{i(\langle\xi, \tau\rangle-\langle\theta, t\rangle)} d t d \xi \\
& =g(\theta, \tau) \\
& =\widehat{c}_{1,3}(\theta, \tau,-\theta, \tau) \phi(\theta, \tau)
\end{aligned}
$$

Finally $E|\widetilde{A}(\theta, \tau)-A(\theta, \tau) \phi(\theta, \tau)|^{2}=0 \quad \forall(\theta, \tau) \in \mathbb{R}^{2 d}$ follows from (41), (76) and (78), and hence (67) is proved.

The formula (66) together with Theorem 6.2 are useful for optimization of the function $\Phi$ for estimation of $W_{E}$ using $W * \Phi$. This problem will be treated in a subsequent paper.

Acknowledgments. The author would like to thank Professor Wolfgang Hörmann for valuable comments, and the referees for useful suggestions.

\section{References}

[1] J. L. Doob , Stochastic Processes, Wiley, 1953.

[2] H. G. Feichtinger, On a new Segal algebra, Monatshefte für Mathematik, 92 (1981), 269-289. 
[3] H. G. Feichtinger and G. Zimmermann, A Banach space of test functions for Gabor analysis, in Gabor analysis and algorithms - theory and applications (eds. H. G. Feichtinger and T. Strohmer), Birkhäuser, 123-170, 1998.

[4] P. Flandrin, Time-Frequency/Time-Scale Analysis, Academic Press, 1999.

[5] G. B. Folland, Harmonic Analysis in Phase Space, Princeton University Press, 1989.

[6] T. L. Grettenberg, A representation theorem for complex normal processes, IEEE Trans. Info. Theory, 11 (1965), 305-6.

[7] K. Gröchenig, Foundations of Time-Frequency Analysis, Birkhäuser, 2001.

[8] C. W. Helstrom, Probability and Stochastic Processes for Engineers, Maxwell Macmillan, 1991.

[9] E. Hille and R. S. Phillips, Functional Analysis and Semi-Groups, American Mathematical Society, 1957.

[10] W. Hörmann, Generalized stochastic processes and Wigner distribution, $\mathrm{PhD}$ thesis, Universität Wien, 1989.

[11] B. Keville (2003), Multidimensional second order generalised stochastic processes on locally compact abelian groups, $\mathrm{PhD}$ thesis, University of Dublin.

[12] M. Loève, Probability Theory, Van Nostrand, 1963.

[13] W. Martin, Time-frequency analysis of random signals, Proc. ICASSP82, (1982), 1325-1328.

[14] W. Martin and P. Flandrin, Wigner-Ville spectral analysis of nonstationary processes, IEEE Trans. ASSP, 33 (1985), 1461-1470.

[15] K. S. Miller, Complex Stochastic Processes, Addison-Wesley, 1974.

[16] M. M. Rao, Harmonizable processes: structure theory, L'Enseign. Math., 28 (1982), 295-351.

[17] W. Rudin, Fourier Analysis on Groups, Wiley, 1962.

[18] A. M. Sayeed and D. L. Jones, Optimal kernels for nonstationary spectral estimation, IEEE Trans. Sig. Proc., 43 (1995), 478-491.

Department of Electroscience

Lund University

Box 118, SE-22100 Lund

Sweden

(E-mail : pw@es.lth.se) 


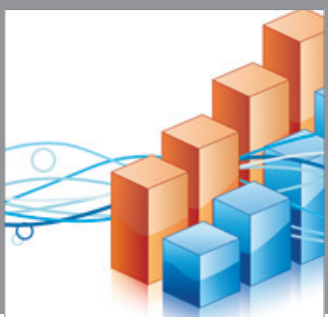

Advances in

Operations Research

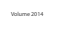

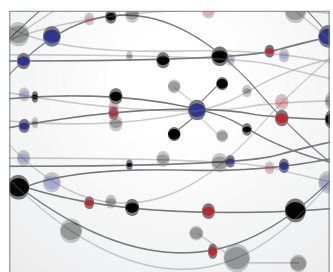

\section{The Scientific} World Journal
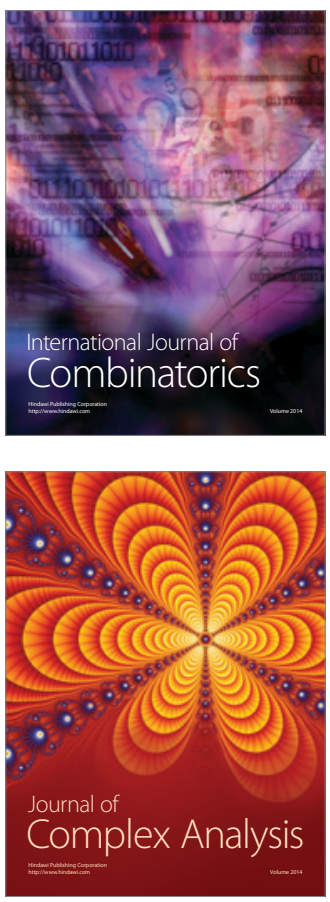

International Journal of

Mathematics and

Mathematical

Sciences
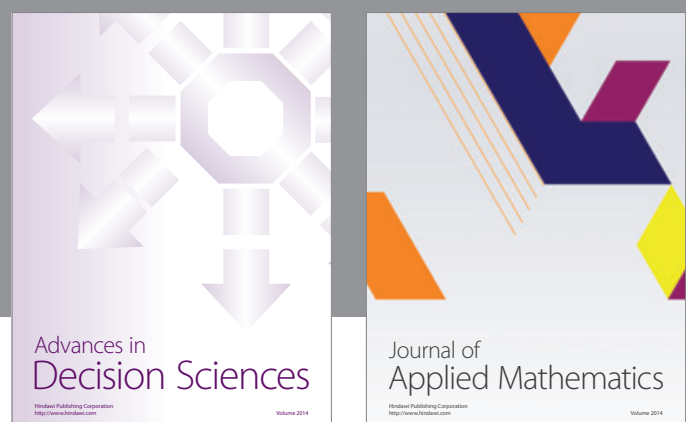

Journal of

Applied Mathematics
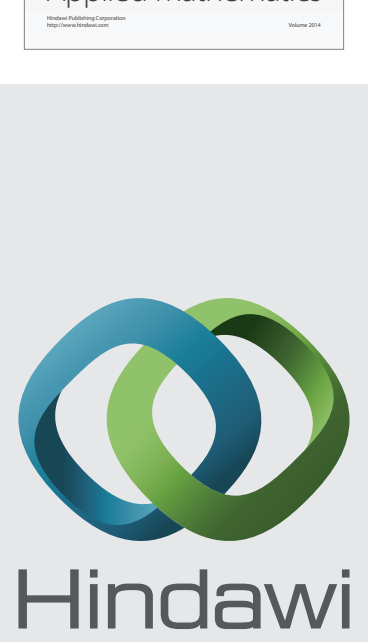

Submit your manuscripts at http://www.hindawi.com
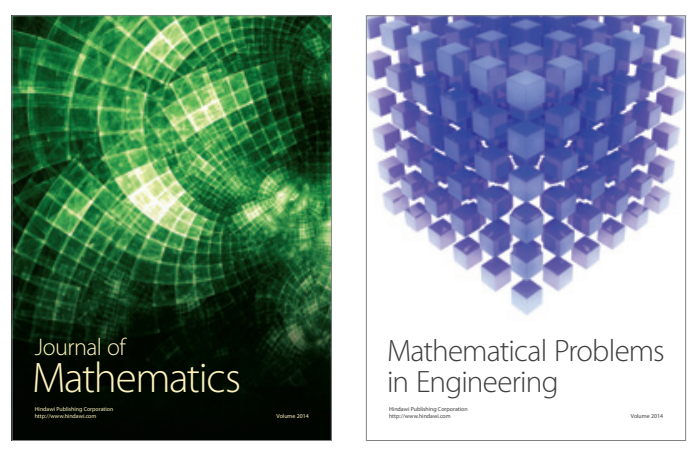

Mathematical Problems in Engineering
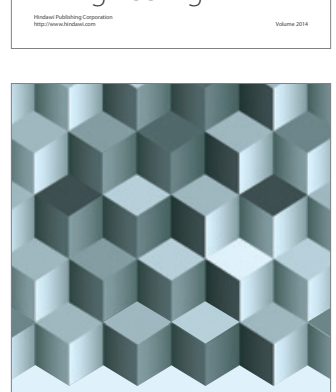

Journal of

Function Spaces
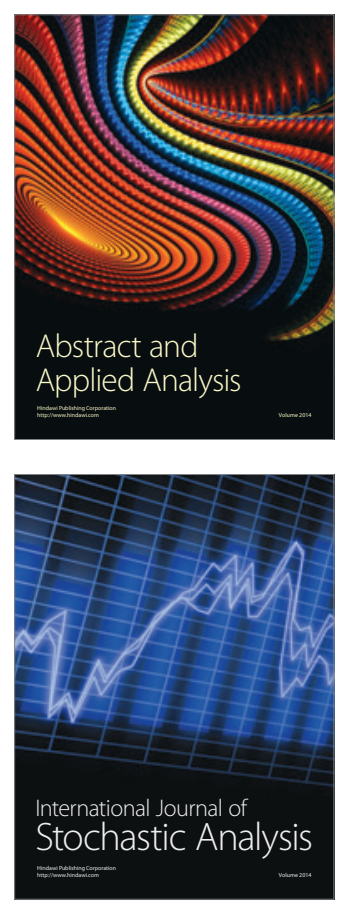

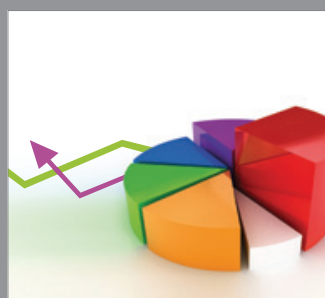

ournal of

Probability and Statistics

Promensencen
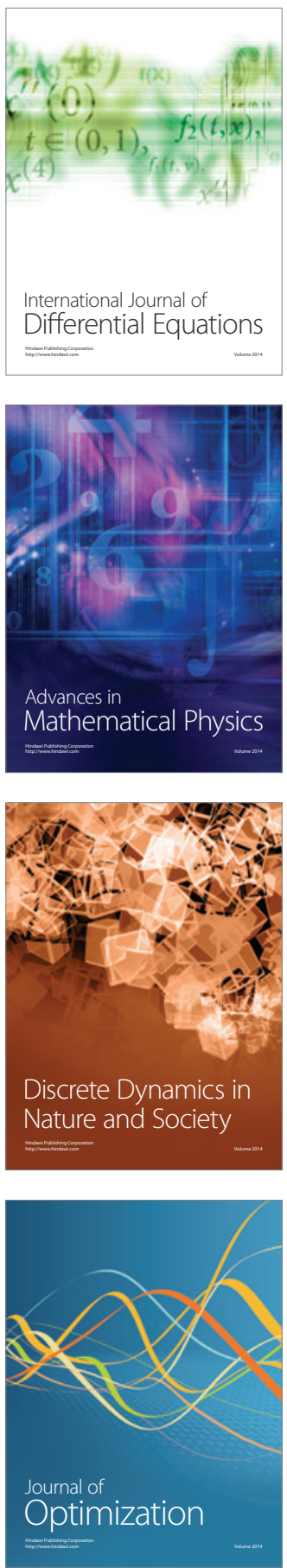